\title{
PROCESS AND SYSTEMS An evaluation of a price transparency intervention for two commonly prescribed medications on total institutional expenditure: a prospective study
}

\author{
Authors: Tessa Langley, ${ }^{\mathrm{A}}$ Julia Lacey, ${ }^{\mathrm{B}}$ Anthony Johnson, ${ }^{\mathrm{C}}$ Clive Newman, ${ }^{\mathrm{D}}$ Deepak Subramanian, ${ }^{\mathrm{E}}$ Milind Khare, ${ }^{\mathrm{F}}$ \\ Rob Skelly, ${ }^{\mathrm{G}}$ Mark Norwood, ${ }^{\mathrm{H}}$ Nigel Sturrock ${ }^{\mathrm{I}}$ and Andrew W Fogarty ${ }^{ }$
}

Providing feedback on cost has been demonstrated to decrease drug demand from clinicians. We conducted a prospective study with a step-wise intervention to test the hypothesis that providing information on the cost of drugs to clinicians would modify total expenditure. Participants included individuals who were admitted to the Royal Derby Hospital from November 2013 to November 2015 under the care of physicians. The cost of all antibiotics and inhaled corticosteroids was added to the electronic prescribing system. The main outcome was the weekly cost for antibiotics and inhaled corticosteroids in the intervention period compared to baseline costs. Mean weekly expenditure on antibiotics per patient decreased by $€ 3.75$ ( $95 \%$ confidence intervals $[\mathrm{CI}]-6.52$ to -0.98 ) after the intervention from a pre-intervention mean of $£ 26.44$, and then slowly increased subsequently by $€ 0.10 /$ week $(95 \% \mathrm{CI}+0.02$ to +0.18$)$. Mean weekly expenditure on inhaled corticosteroids per patient did not substantially change after the intervention ( $-£ 0.03,95 \%$ CI -0.06 to -0.01 after the intervention from a pre-intervention mean of $£ 5.29$ per person). New clinical guidelines for inhaled corticosteroids were associated with a decrease in weekly expenditure, but provision of feedback on drug costs resulted in no sustained change in institutional expenditure. However, clinical guidelines have the potential to modify clinical prescribing behaviour.

\section{Introduction}

Increasing demand for healthcare has led to a need for strategies to rationalise unnecessary demand, without reducing the quality of healthcare provision. There is variation in the cost of treating similar conditions between individual clinicians, ${ }^{1}$ which may

Authors: Aassociate professor, University of Nottingham, Nottingham, UK; ${ }^{\text {B }}$ pharmacist, Royal Derby Hospital, Derby, UK; ${ }^{\text {C }}$ pharmacy technician, Royal Derby Hospital, Derby, UK; D pharmacist, Royal Derby Hospital, Derby, UK; Econsultant, Royal Derby Hospital, Derby, UK; F consultant, Royal Derby Hospital, Derby, UK; ${ }^{G}$ consultant, Royal Derby Hospital, Derby, UK; ${ }^{H}$ director of informatics, Hospital HQ, Royal Derby Hospital, Derby, UK; I medical director, Hospital HQ, Royal Derby Hospital, Derby, UK; ' reader in clinical epidemiology, University of Nottingham, Nottingham, UK represent a degree of suboptimal usage of healthcare resources. This is probably because physicians' choice of medication is predominantly determined by heterogeneous past clinical experiences as well as by local and national guidelines.

Behavioural insight theory suggests that knowledge of psychological processes may be utilised to design interventions that enable doctors to make more informed decisions. ${ }^{2,3}$ This approach is light touch, with no element of obligation for those who are happy with their current practice. An example of this approach is the provision of extra information to clinicians, with no intention of directing the clinician in their decision making (potentially reducing their autonomy and clinical obligations to the patient), but simply to inform them of the cost of this decision. We have previously added the cost of a commonly used blood test to the reports that clinicians receive and observed a $32 \%$ decrease in demand over 12 months. ${ }^{4}$ Testing this approach in other areas of healthcare is important, as it is low-cost and easily scalable if demonstrated to be effective in modifying demand safely.

The purpose of the current study was to extend this cost-feedback approach to prescription drugs in a hospital setting. Specifically, we used electronic prescribing software to provide the cost of all antibiotics and corticosteroid inhalers to clinicians, and evaluated the impact of this intervention using a prospective study design. These interventions were selected as there are a number of options in each therapeutic category available to prescribers, and hence this permitted testing of the hypothesis that provision of cost information will modify the choice of medication. During the period of this prospective study, new clinical guidelines were introduced to help physicians in their choice of inhaled corticosteroids. As a consequence, we were also able to explore the efficacy of a different non-nudge intervention on clinical decision making.

\section{Methods}

Study population

The study population consisted of all individuals who were admitted to the Royal Derby Hospital (RDH) and discharged by the medical general physicians who care for adults between November 2013 and November 2015. The RDH is a busy acute medical hospital that admitted 140,960 individuals in 2014. The study was an evaluation of a health service modification and no ethical approval was required. The study was designed as a 


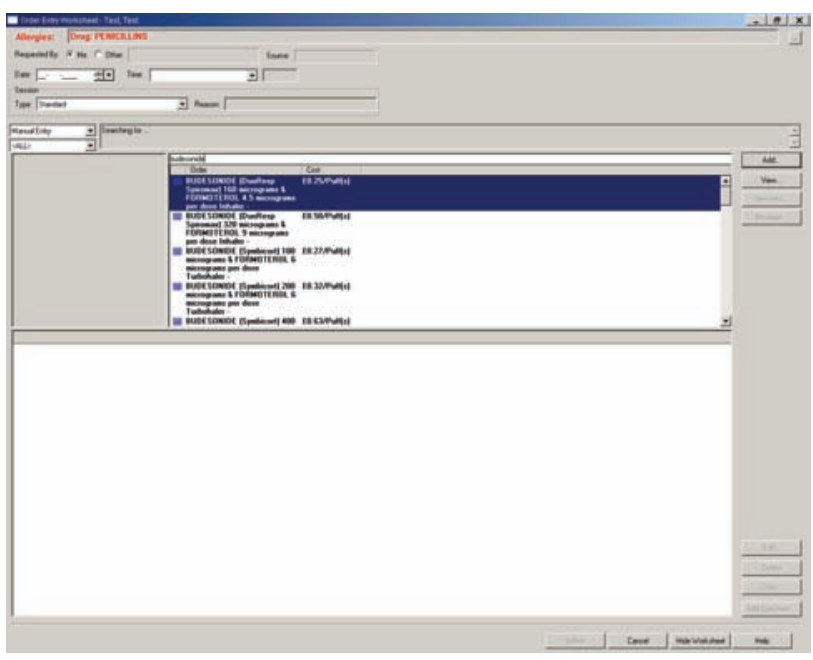

Fig 1. Example of drug costing on the electronic prescribing system.

single intervention that was added to the existing framework of healthcare professionals (including microbiologists and pharmacists) that support clinical decision making.

\section{Intervention}

The RDH uses iSoft electronic prescribing and administration software to permit electronic prescribing of drugs. This has a setting that permits the cost of the drug to be added to the display that the prescribing clinician sees immediately prior to selecting the medication of choice. The prescribing clinician only sees the cost of the antibiotic or inhaled corticosteroid that is selected (as demonstrated in Fig 1). To provide a comprehensive list of medications, costs for every antibiotic (cost per day) and inhaled corticosteroid (cost per puff) listed in the March-September 2014 edition of the British National Formulary were provided by the RDH pharmacy department, including adjustment for the extra costs of administering intravenous drugs. ${ }^{5}$ The intervention was implemented on the 8 November 2014

During the period of the study, generic inhaled corticosteroids became available at the RDH during the first 6 months of 2015 , and new guidelines for the use of inhaled corticosteroids by clinicians were independently introduced by the respiratory medicine department in May/June 2015. These guidelines promoted the use of the cheaper generic medications where possible. This change became evident when the weekly cost data were analysed (Fig 2), and it was evident that an external change had modified inhaled corticosteroid usage in the institution.

\section{Data}

The data collection period spanned 11 November 2013 to 2 November 2015. Weekly data on all prescribed drugs were collected from the electronic prescribing system along with the number of individuals who were prescribed those drugs. The data from the preceding 52 weeks were compared with the 52 weeks after the intervention was implemented.

The prescribing data were combined with the data on drug costs to calculate the total weekly expenditure on antibiotics and inhaled corticosteroids. Data on the total weekly numbers of patients in both therapeutic categories (those prescribed antibiotics and inhaled corticosteroids) were also collected to permit adjustment for clinical activity in the statistical analysis by estimating a cost per patient.

\section{Statistical analysis}

We used segmented regression analyses to evaluate the effect of the intervention on a) total weekly expenditure on antibiotics
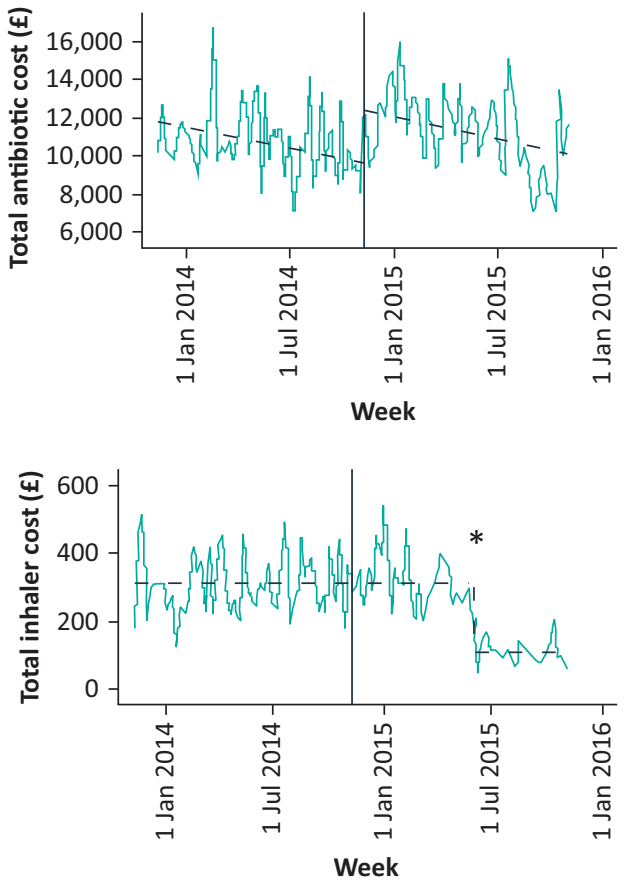
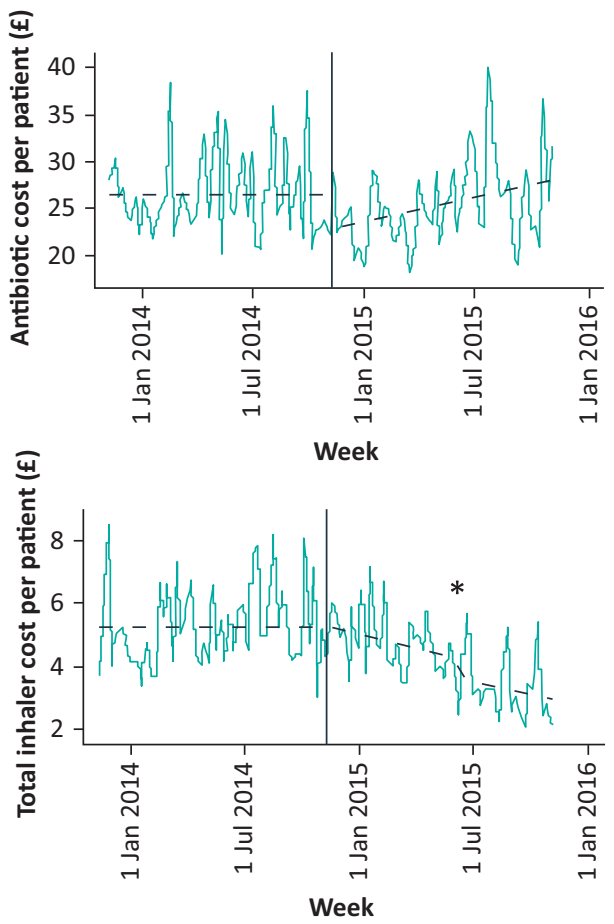

Fig 2. Change in the cost of total antibiotics and total inhalers per patient per week. Vertical lines represent the cost-feedback intervention. *Represents the introduction of the inhaled corticosteroid protocol. 
Table 1. Segmented regression analysis of drug-cost data

\begin{tabular}{|c|c|c|c|c|c|}
\hline & $\begin{array}{l}\text { Pre-intervention } \\
\text { mean (unadjusted) }\end{array}$ & $\begin{array}{l}\text { Post-intervention } \\
\text { mean (unadjusted) }\end{array}$ & $\begin{array}{l}\beta 1 \text { - baseline } \\
\text { trend }(95 \% \mathrm{CI})\end{array}$ & $\begin{array}{l}\beta 2 \text { - step level } \\
\text { change }\end{array}$ & $\begin{array}{l}\beta 3 \text { - change in } \\
\text { trend }\end{array}$ \\
\hline $\begin{array}{l}\text { Total spend on anitbiotics per } \\
\text { week }(£)\end{array}$ & 10,719 & 11,260 & $\begin{array}{l}-43.58(-43.58 \\
\text { to }-19.60) \\
p<0.001\end{array}$ & $\begin{array}{l}+2,807.50 \\
(+1,367.83 \text { to } \\
+4,247.07) \\
p<0.001\end{array}$ & - \\
\hline $\begin{array}{l}\text { Antibiotics spend per patient } \\
\text { per week }(£)\end{array}$ & 26.44 & 25.40 & - & $\begin{array}{l}-3.75(-6.52 \text { to } \\
-0.98) p=0.008\end{array}$ & $\begin{array}{l}+0.10(+0.02 \text { to } \\
+0.18) p=0.015\end{array}$ \\
\hline $\begin{array}{l}100 \text { mg oral doxycycline } \\
\text { capsules spend per patient per } \\
\text { week ( } € \text { ) }\end{array}$ & 0.020 & 0.023 & - & $\begin{array}{l}+0.003(+0.001 \\
\text { to }+0.005) \\
p<0.001\end{array}$ & - \\
\hline Total inhaler spend per week (£) & 309.29 & 227.74 & - & - & - \\
\hline $\begin{array}{l}\text { Inhaler spend per patient per } \\
\text { week }(£)\end{array}$ & 5.29 & 4.17 & - & - & $\begin{array}{l}-0.03(-0.06 \text { to } \\
-0.01) p=0.11\end{array}$ \\
\hline \multicolumn{6}{|c|}{$\begin{array}{l}1: \text { weekly change in spending before intervention. } \\
\beta 2: \text { step change in weekly spend after intervention. } \\
\beta 3 \text { : absolute change in trend in weekly spend after intervention, compared with baseline trend. } \\
\beta 1+\beta 3=\beta 4 \text { post-intervention slope. } \\
\text { Inhaler models include a dummy variable to adjust for a change in local prescribing policy. Only statistically significant variables are included in the parsimonious } \\
\text { models. Gaps in the table reflect that variables were not significant and were not included in the final model. } \\
\mathrm{CI}=\text { confidence interval }\end{array}$} \\
\hline
\end{tabular}

and inhalers and b) weekly per patient expenditure on antibiotics and inhalers. The impact on oral doxycycline capsules was also modelled, as this drug is a relatively cheap but commonly used treatment for respiratory infection. We hypothesised that the frequency of doxycycline prescription would increase once prescribing costs were made available to physicians. Segmented regression is a powerful interrupted time series method that can identify whether an intervention introduced at a single, known point in time had an immediate or delayed impact on the outcome measure and whether any impact that did occur had a transient or long-term effect over time. ${ }^{6}$

Parsimonious models were identified by backward elimination, dropping any parameters that were not significant at the $5 \%$ significance level. ${ }^{6,7}$ All of the parsimonious models were checked to see whether there was any autocorrelation, ie correlation between successive observations, using the autocorrelation function (ACF), which plots the residuals from the segmented regression analysis. Stata software (Texas, USA) was used for the statistical analysis.

\section{Results}

Over the study period, the mean numbers of individuals who were prescribed antibiotics and corticosteroids were 428 and 55 individuals per week, respectively. The total weekly expenditure per patient for both antibiotics and inhalers over the period of study is shown in Fig 2. The mean weekly expenditure on antibiotics per patient was $£ 26.44$ in the baseline period and $£ 25.40$ in the post-intervention period (Table 1). Comparable expenditure for inhaled corticosteroids was also lower in the post-intervention period ( $₫ 4.17$ per patient per week) compared to that in the baseline period ( $₫ 5.29$ per patient per week). This decrease in the weekly cost of inhaled corticosteroids was largely due to a change in local prescribing policy from June 2015 onwards and further analysis of this period is therefore adjusted for this event by fitting a dummy variable in the inhaler models.
The results of the segmented regression analysis are presented in Table 1. These data allow more detailed analysis of the time course of changes in expenditure after the intervention was implemented. Per patient expenditure on antibiotics decreased immediately after the intervention (by $£ 3.75$ per patient per week or $14 \%$ of baseline expenditure, $p=0.008$ ), but this decrease was followed by a trend of increasing expenditure ( $£ 0.10$ per patient week or $0.4 \%$ of baseline, $p=0.015$ ). There was an immediate increase in the weekly data for frequency of prescription of doxycycline capsules, a cheaper oral antibiotic, of 18\% (confidence intervals [CI] 15 to 26) from a baseline of 1.78p per patient per week after the intervention (Table 1, Fig 3).

For both overall inhaler spend and inhaler spend per patient, there was no statistically significant underlying trend and no

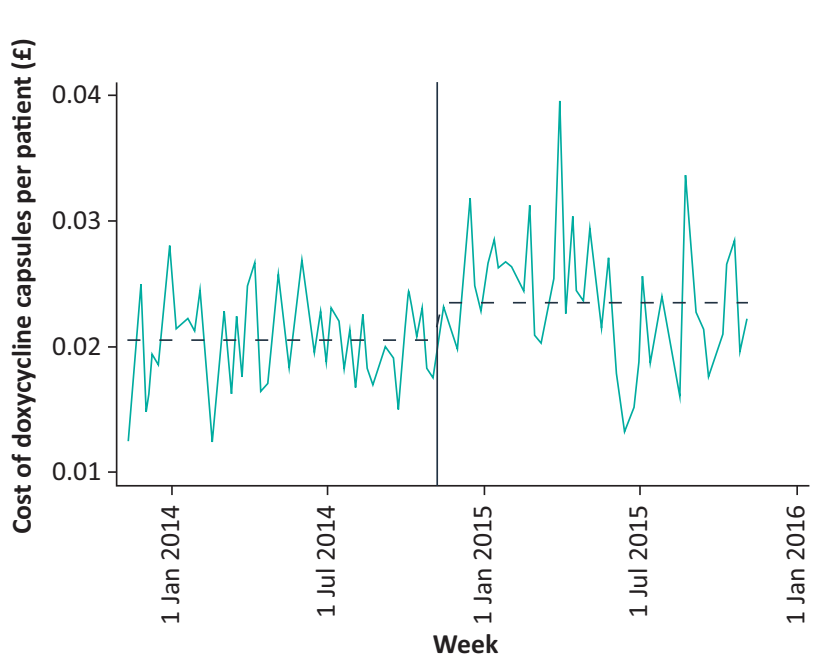

Fig 3. Change in the cost of doxycycline capsules per patient per week. Vertical line represents the cost-feedback intervention. 
change in trend post-intervention. There was a small change in trend in spending on inhalers, such that weekly per patient spend was decreasing by $3 p$ per week. Following a change in local prescribing policy in June 2015, the drop in weekly expenditure on inhalers was $58 \mathrm{p}$ for per patient spend and $£ 203$ for total inhaler spend per week (representing a 65\% drop from an unadjusted mean of $£ 311$ per week in the period prior to the local policy change).

\section{Conclusions}

This is the first prospective study to explore the impact of providing the cost of antibiotics and inhaled corticosteroids to clinicians in a busy acute medical hospital in the UK. The intervention led to an immediate increase of $18 \%$ in prescriptions for doxycycline, which is a relatively cheap option when treating respiratory infection, but this did not translate into a sustained decrease in overall weekly expenditure on antibiotics, which was the primary outcome of interest. There was no substantial change in expenditure on inhaled corticosteroids that could be attributed to the costfeedback intervention, but a change in local clinical protocols for inhaled corticosteroids that was independent of our intervention and which promoted generic alternatives was associated with a decrease in weekly expenditure on these medications.

By adding the costs of the drugs of interest so that they were clearly visible to the prescribing clinician, we can be confident that the intervention was implemented successfully, as it was impossible to prescribe the drugs without seeing the price once the intervention was in place (Fig 1). Our use of total institutional expenditure on medications as the primary outcome measure is a strength and this represents a composite macroeconomic measure that is pertinent to efficient healthcare delivery. A further strength of the study was our ability to collect electronic data on all drugs prescribed in the $\mathrm{RDH}$, thus providing assurance that we obtained complete data on prescribing activity. By studying this population over a period of one year before and after the intervention, we can be confident that seasonal variation does not confound our analysis. Studying for a longer period is unlikely to be helpful as demographic changes may begin to impact a study population as the study is extended and as we are unable to adjust for disease severity. Our intervention has one limitation which is that the costs of the drugs are only visible once that drug has been selected, and not alongside other therapeutic options. Hence, our intervention provides information about the cost of the selected medication, but not an immediate comparison with cost of alternative drugs. Provision of direct cost comparisons between drugs is not a practical option as the decision to prescribe the medication is generally made at the patient's bedside and the prescribing is done subsequently, often elsewhere. This division of workload may contribute to the absence of any substantial sustained impact on total medication selection, as the person who makes the decision may not be the person who prescribes the drug and hence is exposed to the cost information. In addition, there are many antibiotics that can be selected to treat a clinical scenario, and comparing them all while attending to the demands of a busy healthcare setting is not a feasible option.

It is surprising that there are no prior studies of the impact of providing pricing information for commonly used medications on clinician decision making. This is probably a consequence of the challenge of rigorously evaluating change within healthcare settings, where many factors can impact on the demand for healthcare and the 'system' remains in constant dynamic flux. As a consequence, our choice of the before/after or step-change regression model is probably the optimal study design for use in the 'real world' of applied health services research. Other options, such as the randomised controlled trial, are either not appropriate or not deliverable in this context. ${ }^{8}$ By knowing the exact date of the intervention, we were able to assess the change in prescribing patterns in the following weeks. The $18 \%$ increase in the prescribing of doxycycline immediately after our cost-pricing intervention was implemented is evidence that there was a change in physician behaviour. This is a large increase in the selection of a cheaper but effective antibiotic option that did not occur by chance, and it provides evidence that our drug-cost information was observed by the prescribing doctors. This is important as it shows that the clinicians' later lack of response to cost information was clearly not a consequence of the intervention not being seen. It is also important to note that this is pragmatic health services research that tested a simple hypothesis and was delivered with a limited budget. As a consequence, we were unable to follow up individual clinicians to ask what they thought of the intervention. As with any new intervention, the introduction of cost information underwent a risk analysis to ensure that no harm was inadvertently delivered. There were no adverse events reported and future studies in this area should also consider similar measures.

The impact of adding price transparency for antibiotic costs on demand was biphasic. There was an initial immediate decrease of $14 \%$ from baseline in cost per patient per week, but this was followed by a small weekly increase in costs over the following 12 months of $0.4 \%$ of the baseline value per week. This represents a reversal of the initial effect that we are unable to explain, but is an important factor in the consideration of these data. It is possible that the presentation of the price displays could be optimised and even varied to prevent familiarisation that results in "alert blindness', and that this may improve awareness of the cost 'message', although we were constrained by the presentational options available in the electronic prescribing software. As can be seen from Fig 1, our cost data were clearly available to the prescribing clinician. Alternative strategies to optimise the display of cost information could be considered in the future, but it is important to remember that the primary purpose of electronic prescribing software is the safe prescribing of medications, and we did not want our cost feedback to detract from this. In addition, we were unable to deliver qualitative research to assess clinicians' perspectives on the provision of healthcare costs due to budgetary constraints.

Consideration of the context of this study is important in interpreting the generalisability of the results. The study was set in a busy teaching hospital where clinicians assess and treat patients according to their clinical need, and the patient does not make a payment to contribute to their healthcare costs. Hence, while our observations may be applicable to similar nationally funded healthcare systems, they may not be so generalisable to healthcare systems in which healthcare management decisions and hence costs may also be influenced by the patients' ability to pay or in which medications are rationed as a consequence of scarcity or economic necessity.

This work builds on our previous study, which demonstrated that adding the institutional cost to C-reactive protein results was associated with a $32 \%$ decrease in demand over 52 weeks, ${ }^{4}$ 
although this was not observed in recent data from the USA. ${ }^{9}$ We are not aware of any comparable studies that have prospectively studied the impact of cost information for commonly used drugs on the subsequent selection of these drugs by clinicians. Conventional economic theory suggests that most individuals are influenced to some degree by costs, but applying this theory to medical decision making is challenging and controversial. This is because a physicians' choice of antibiotic is predominantly determined by clinical experience as well as by local guidelines that already take into consideration both local patterns of microbial resistance to antibiotics and cost. Many established antibiotics have similar institutional costs, and the mode of delivery (oral versus intravenous) can be an important in determining total cost. Although the frequency of stepping down from intravenous antibiotics to oral antibiotics does vary depending on the day of the week ${ }^{10}$ (and hence medical staffing levels), our intervention had no effect on the total costs of antibiotics. Control of inappropriate antibiotic prescribing continues to be a priority area because of the need to reduce complications such as Clostridium difficile gastrointestinal infection, and it is possible that the use of antibiotics in this hospital has reached a level where there is limited scope for improvement. Hence, these findings are probably generalisable to institutions with strong existing antibiotic guidelines that are actively promoted and supervised.

By evaluating our cost-information intervention over a period during which a change in clinical guidelines for the use of inhaled corticosteroids also took place, we were inadvertently able to observe a natural experiment in which the impact of one passive 'nudge' intervention (cost-feedback) could be compared with a second more directive approach (clinical guidelines). We are cautious in our interpretation of these data which represents a post hoc analysis that is derived from a necessity to explain the step-change decrease in weekly expenditure on inhaled corticosteroids data rather than an a priori hypothesis. However, the implementation of new prescribing guidelines for inhaled corticosteroids does appear to be associated with a substantial decrease in the costs of prescribing these medications and supports the use of local protocols to promote efficient prescribing.

\section{Key points}

\section{Question}

Does the provision of healthcare costs influence clinicians' prescribing choices for antibiotics and inhalers?

\section{Findings}

Provision of cost information resulted in a transient, non-sustained drop in weekly institutional spending on antibiotics and no change in inhaled corticosteroids expenditure. The introduction of new clinical prescribing guidelines for inhaled corticosteroids was associated with a substantial decrease in weekly costs for this medication category.

\section{Meaning}

Provision of cost information at the point of prescribing had no sustained impact on weekly antibiotic or inhaled corticosteroid costs. The introduction of new clinical guidelines for inhaled corticosteroids during the study period was associated with a drop in weekly expenditure for these medications. This approach may have more leverage in modifying clinical decision-making than the provision of cost-feedback.

\section{Competing interests}

The authors declare no competing interests.

\section{References}

1 Kennedy P, Leathley C, Hughes C. Clinical practice variation. Med J Aust 2010;193:S97-9.

2 Perry C, Chhatralia K, Damesick D, Hobden S, Volpe L. Behavioural insights in health care. The Health Foundation, 2015. www.health. org.uk/sites/health/files/BehaviouralInsightsInHealthCare.pdf [Accessed 29 December 2017].

3 Thaler R, Sunstein C. Nudge. London: Penguin, 2008.

4 Fogarty A, Sturrock N, Premji K, Prinsloo P. Hospital clinicians' responsiveness to assay cost feedback: a prospective blinded controlled intervention study. JAMA Intern Med 2013;173:1654-5.

5 Zanten A, Engelfriet P, Dillen K et al. Importance of nondrug costs of intravenous antibiotic therapy. Critical Care 2003;7:R184-90.

6 Wagner AK, Soumerai SB, Zhang F, Ross-Degnan D. Segmented regression analysis of interrupted time series studies in medication use research. J Clin Pharm Ther 2002;27:299-309.

7 Penfold RB, Zhang F. Use of interrupted time series analysis in evaluating health care quality improvements. Acad Pediatr 2013;13:38-44.

8 Venkataramani A, Bor J, Jena A. Regression discontinuity designs in healthcare research. BMJ 2016;352:i1216.

9 Sedrak M, Myers J, Small D et al. Effect of a price transparency intervention in the electronic health record on clinician ordering of inpatient tests: the PRICE randomized clinical trial. JAMA Intern Med 2017:177:939-45.

10 Lewis S, Langley T, Lacey ] et al. Frequency of stepping down antibiotics and nebuliser treatment is lower at weekends compared to weekdays: an observational study. Clin Med 2018;17:504-7.

Address for correspondence: Dr Andrew Fogarty, University of Nottingham, Nottingham NG5 1PB, UK. Email: andrew.fogarty@nottingham.ac.uk 